\title{
Neurosurgical intervention in patients with mild traumatic brain injury and its effect on neurological outcomes
}

\author{
Kevin James Tierney, MD, ${ }^{1}$ Natasha V. Nayak, MD, ${ }^{2,4}$ Charles J. Prestigiacomo, MD, ${ }^{3}$ and \\ Ziad C. Sifri, MD²
}

Departments of ${ }^{1}$ Emergency Medicine, ${ }^{2}$ Surgery, and ${ }^{3}$ Neurological Surgery, Rutgers New Jersey Medical School, Newark, New Jersey; and ${ }^{4}$ Department of Ophthalmology, New York Eye and Ear Infirmary of Mount Sinai, New York, New York

\begin{abstract}
OBJECTIVE The object of this study was to determine the mortality and neurological outcome of patients with mild traumatic brain injury (mTBI) who require neurosurgical intervention (NSI), identify clinical predictors of a poor outcome, and investigate the effect of failed nonoperative management and delayed NSI on outcome.
\end{abstract}

METHODS A cross-sectional study of 10 years was performed, capturing all adults with mTBI and NSI. Primary outcome variables were mortality and Glasgow Outcome Scale (GOS) score. Patients were divided into an immediate intervention group, which received an NSI after the initial cranial CT scan, and a delayed intervention group, which had failed nonoperative management and received an NSI after 2 or more cranial CT scans.

RESULTS The mortality rate in mTBI patients requiring NSI was $13 \%$, and the mean GOS score was $3.6 \pm 1.2$. An age $>60$ years was independently predictive of a worse outcome, and epidural hematoma was independently predictive of a good outcome. Logistic regression analysis using independent variables was calculated to create a model for predicting poor neurological outcomes in patients with $\mathrm{mTBI}$ undergoing NSI and had $74.1 \%$ accuracy. Patients in the delayed intervention group had worse mortality ( $25 \%$ vs $9 \%)$ and worse mean GOS scores $(2.9 \pm 1.3$ vs $3.7 \pm 1.2)$ than those in the immediate intervention group.

CONCLUSIONS Data in this study demonstrate that patients with $\mathrm{mTBI}$ requiring NSI have higher mortality rates and worse neurological outcomes and should therefore be classified separately from mTBI patients not requiring NSI. Additionally, mTBI patients requiring NSI after the failure of nonoperative management have worse outcomes than those receiving immediate intervention and should be considered separately.

http://thejns.org/doi/abs/10.3171/2015.4.JNS142440

KEY WORDS mild traumatic brain injury; Glasgow Outcome Scale; mortality; elderly

$\mathrm{T}$ RaUMATIC brain injury is a major health concern in the United States, with an incidence of more than 1.5 million cases per year, resulting in greater than 50,000 annual deaths. ${ }^{17}$ Of these annual cases, $70 \%-90 \%$ are classified as mild traumatic brain injuries (mTBIs), ${ }^{4}$ defined by a Glasgow Coma Scale (GCS) score $>12$. The literature suggests that between $0.7 \%$ and $8.7 \%$ of patients with mTBI will require neurosurgical intervention (NSI). 1,2,6,7,9,10,20,22 The majority of TBIs, and mTBIs more specifically, are treated nonoperatively; a small number of injuries require surgical intervention. Nonoperative management includes observation, serial neurological checks, and medical management. Surgical intervention in TBI focuses on removing a localized insult to the brain and is feasible when there is either a depressed skull fracture or a localized mass lesion. ${ }^{3}$

Patients with mTBI requiring NSI are a small but un- derstudied population. To help guide patient counseling and the appropriate utilization of acute care, hospital, and rehabilitation resources, it is important to understand the outcomes of the mTBI population in particular. While the mortality rate for mTBI is less than $2 \%, 6,8,10$ the mortality rate for mTBI patients who require NSI is unknown. No study to date has focused on the Glasgow Outcome Scale (GOS) score and mortality rates for mTBI patients requiring NSI. Additionally, outcome differences between those who receive immediate NSI and those who receive delayed NSI after the failure of nonoperative management are also unknown.

The purpose of this study was to 1) determine the outcome of patients with mTBI who received an NSI, 2) identify clinical predictors of a poor outcome, and 3) investigate the effect of delayed NSI on outcome.

ABBREVIATIONS AIS = Abbreviated Injury Score; GCS = Glasgow Coma Scale; GOS = Glasgow Outcome Scale; ICB = intracranial bleed; ISS = Injury Severity Score; $\mathrm{LOS}=$ length of stay; $\mathrm{mTBI}=$ mild traumatic brain injury; $\mathrm{NSI}$ = neurosurgical intervention.

SUBMITTED October 25, 2014. ACCEPTED April 2, 2015.

INCLUDE WHEN CITING Published online September 25, 2015; DOI: 10.3171/2015.4.JNS142440. 


\section{Methods}

We conducted a cross-sectional study of adults (age > 17 years) with mTBI who, between 2001 and 2010, had been admitted to the University Hospital in Newark, New Jersey, a Level I trauma center. Patients were identified in the trauma registry and their medical records were retrospectively reviewed. The study population was mTBI patients requiring NSI, defined as placement of an intracranial pressure monitor, ventriculostomy, craniotomy, craniectomy, cranioplasty, bur holes, or elevation of skull fractures; therefore, patients who only had a measuring device placed were considered to have received an NSI. Patients with a history of prior brain surgery or cerebral pathology (acquired/congenital), chronic neurological conditions such as schizophrenia or Alzheimer disease, and incomplete medical records were excluded from our analysis. Notably, patients on anticoagulants were not excluded.

Demographic information, including patient age, sex, Injury Severity Score (ISS), GCS score, head Abbreviated Injury Score (AIS), and use of antiplatelet or anticoagulant, was collected. The number, date, time, and findings of the initial and all subsequent cranial CT scans were recorded from radiographic reports by staff radiologists. Operative reports on the NSI were reviewed for timing and type of operation performed.

Outcome variables were reviewed. Primary outcome variables were the GOS score and mortality rate. A good neurological outcome was defined as a GOS score of 4 (mild disability) or 5 (good recovery), whereas a poor neurological outcome was defined as a GOS score of 3 (severe disability), 2 (persistent vegetative state), or 1 (death). ${ }^{11}$ Complications from the NSI, length of hospital stay, and discharge location were noted. The follow-up interval for each patient was the entire duration of their in-hospital stay.

Patients were then subdivided into an initial intervention group and a delayed intervention group. Patients in the immediate intervention group were those who received an

TABLE 1. Demographics in 139 patients with $\mathrm{mTBI}$ requiring NSI ${ }^{*}$

\begin{tabular}{lc}
\hline \multicolumn{1}{c}{ Parameter } & No. \\
\hline Mean age in yrs & $42 \pm 18$ \\
\hline Male (\%) & $117(84)$ \\
\hline Mechanism of injury (\%) & \\
\hline Assault & $45(32)$ \\
\hline Fall & $45(32)$ \\
\hline Motor vehicle accident & $20(14)$ \\
\hline Pedestrian struck & $22(16)$ \\
\hline Other & $7(5)$ \\
\hline Mean ISS & $26 \pm 8$ \\
\hline Mean head AIS & $4.4 \pm 0.6$ \\
\hline Mean GCS score & $14.5 \pm 0.7$ \\
GCS Score 13 (\%) & $16(12)$ \\
GCS Score 14 (\%) & $38(27)$ \\
GCS Score 15 (\%) & $85(61)$ \\
\hline Antiplatelet or anticoagulant use (\%) & $11(8)$ \\
\hline
\end{tabular}

* Mean values are expressed with the standard deviation.
TABLE 2. Cranial CT results in 139 patients*

\begin{tabular}{ll}
\hline \multicolumn{1}{c}{ Finding } & No. $(\%)$ \\
\hline Subarachnoid hematoma & $78(56)$ \\
\hline Epidural hematoma & $54(39)$ \\
\hline Subdural hematoma & $93(67)$ \\
\hline Intraparenchymal hemorrhage & $74(53)$ \\
\hline Skull fracture & $97(70)$ \\
\hline
\end{tabular}

${ }^{*}$ Number of injuries is greater than the number of patients because some patients had multiple injuries.

NSI after an initial cranial CT scan. Patients in the delayed intervention group were those in whom nonoperative management had failed and who had an NSI after 2 or more cranial CT scans.

Patient data were reported together, either as the mean \pm standard deviation or as a percentage. Odds ratios were calculated for factors affecting poor outcome. Logistic regression was used to calculate the adjusted odds ratio for select variables. The data were divided into an initial operative management versus failed nonoperative management, and the data were compared using the independent-samples t-test and the Mann-Whitney U-test. Statistical significance was attributed to a $\mathrm{p}<0.05$. Data were analyzed using Statistical Package for the Social Sciences, version 14 (SPSS Inc.).

This study received approval from the Internal Review Board at the University of Medicine and Dentistry of New Jersey-New Jersey Medical School.

\section{Results}

During the study period of January 2001 through December 2010, 1688 adults with mTBI presented to the hospital. Of these patients, 179 were identified as having had an NSI. Forty patients were excluded based on the above exclusion criteria. Subsequently, 139 patients $(8 \%)$ met our study inclusion criteria. Their mean age was 42 years, and $84 \%$ of the patients were male. The most prevalent mechanisms of injury were fall and assault. The mean GCS score was $14.5 \pm 0.7$, with $61 \%$ of subjects presenting with a GCS score of 15 . The mean ISS was $26 \pm 8$, and the mean AIS was $4.4 \pm 0.6$. Additionally, chi-square analysis of the AIS and ISS as compared with good and

TABLE 3. Neurosurgical interventions performed in 139 patients

\begin{tabular}{lc}
\hline \multicolumn{1}{c}{ Procedure } & No. (\%) \\
\hline Bur hole & $4(2.9)$ \\
\hline Craniectomy & $12(8.6)$ \\
\hline Cranioplasty & $8(5.8)$ \\
\hline Craniotomy & $96(69.1)$ \\
\hline Elevation of skull fracture & $16(11.5)$ \\
\hline Placement of extraventricular drain & $14(10.1)$ \\
\hline Placement of ICP monitor & $54(38.8)$ \\
\hline
\end{tabular}

*Total percentage is greater than $100 \%$ as multiple patients had multiple procedures performed. 


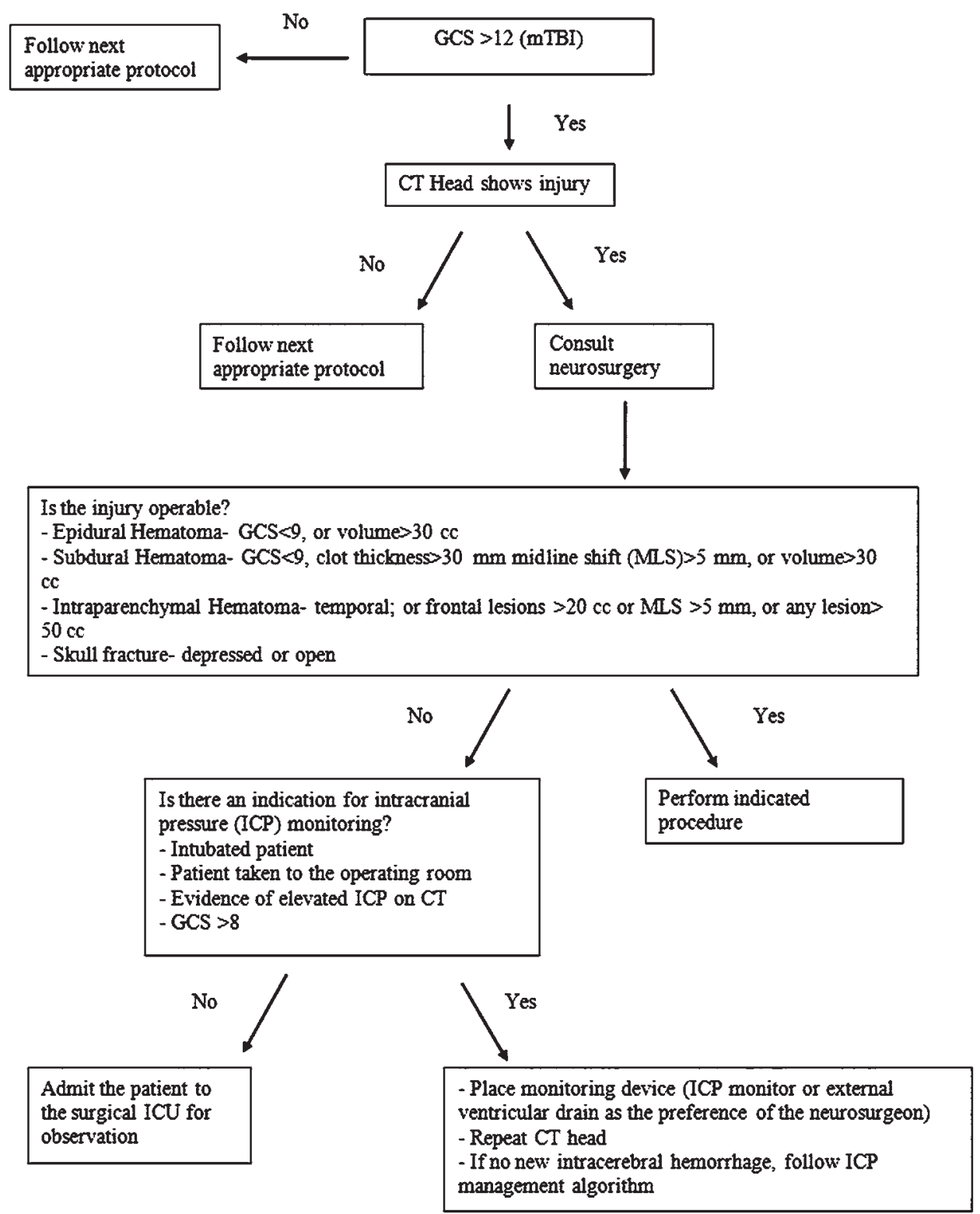

FIG. 1. Flowchart demonstrating how patients with mTBI are managed at the University Hospital in Newark, New Jersey. ICU = intensive care unit.

poor outcomes showed that ISS was not significantly associated with a poor outcome, whereas the AIS was. Eleven patients $(8 \%)$ were on antiplatelet or anticoagulant therapy, which included aspirin, warfarin, clopidogrel, and/or enoxaparin (Table 1).

According to initial cranial CT results (Table 2), $71 \%$ of patients had multiple intracranial bleeds (ICBs), 26\% had 1 ICB, and $4 \%$ had no ICB (1\% with initially normal CT and $1 \%$ with skull fracture). The most prevalent injuries were skull fractures (70\%) and subdural hematomas (67\%), while the least prevalent was epidural hematoma (39\%).

In our study population, the most common NSI was craniotomy (69.1\%; Table 3). The protocol for managing mTBI patients requiring NSI at our trauma center is shown in Figs. 1 and 2, but the ultimate treatment decisions were deferred to the treating neurosurgeon. The overall mortality rate in our study was $13 \%$. The overall rate of complication due to NSI was $14 \%$, with the most common complications being persistently elevated intracranial hypertension (3\%) and brain death (3\%; Table 4). The average length of stay (LOS) was 16 days (Table 5). Forty-four percent of the patients had a poor outcome $(\mathrm{GOS}<4)$. There was no difference in any of the primary outcome measures when comparing GCS scores of 13, 14, and 15.

In patients with an mTBI warranting NSI, factors that increased the odds of a poor neurological outcome included an age $>60$ years, subdural hematomas, subarachnoid hematomas, postoperative complications, delayed NSI, and antiplatelet or anticoagulant use ( $<<0.05$; Table 6). However, when controlling for craniotomy, the delayed in- 


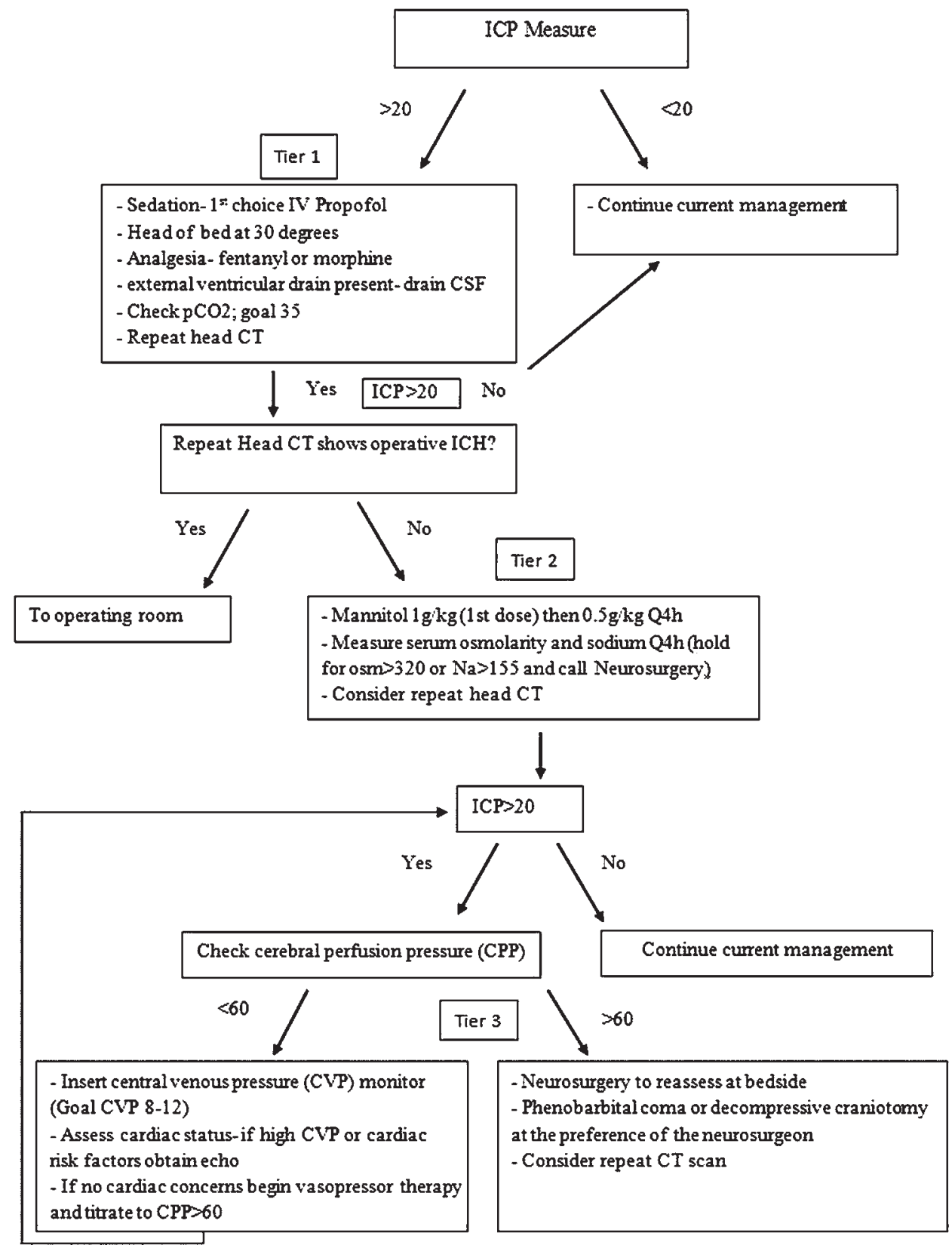

FIG. 2. Flowchart demonstrating how intracranial pressure is managed at the University Hospital in Newark, New Jersey. $h=$ hour(s); IV = intravenous; $Q$ = every.

tervention group no longer significantly increased the odds of a poor neurological outcome (OR 0.9, 95\% CI 0.3-5.4). Conversely, epidural hematomas, skull fractures, and an NSI consisting of craniotomy significantly decreased the odds of a poor neurological outcome $(\mathrm{p}<0.05)$.

Logistic regression analysis was used to create a model for predicting poor neurological outcomes in patients with mTBI treated with an NSI. The model was able to predict a poor neurological outcome $56.1 \%$ and $74.1 \%$ of the time without and with independent variables, respectively (Table 7). The analysis demonstrated that age was the only independent predictor of a poor neurological outcome; epidural hematoma was the only independent predictor of a good neurological outcome.

Patients with mTBI receiving an NSI were divided into the immediate intervention group and a delayed intervention group (failed nonoperative management) and compared. The percent of patients in the immediate intervention group was $77 \%$, or 107 patients. There was no significant difference in demographic characteristics between the 2 intervention groups (Table 8). Radiographically, the immediate intervention group had significantly more skull fractures and midline shifts $(\mathrm{p}<0.05)$. Patients in the immediate intervention group were also significantly $(\mathrm{p}<$ 
TABLE 4. Complications in 139 mTBI patients*

\begin{tabular}{cc}
\hline \multicolumn{1}{c}{ Complication } & No. (\%) \\
\hline None & $119(86)$ \\
\hline Of NSI & $20(14)$ \\
\hline Cerebral vascular accident & $1(1)$ \\
\hline Abscess, infected bone & $2(1)$ \\
\hline Seizures & $3(2)$ \\
\hline Re-bleed & $2(1)$ \\
\hline Need for additional surgery & $1(1)$ \\
\hline Persistently high ICP & $4(3)$ \\
\hline Brain death & $4(3)$ \\
\hline Hydrocephalus & $1(1)$ \\
\hline CSF leakage & $1(1)$ \\
\hline Cerebral edema & $1(1)$ \\
\hline
\end{tabular}

* Number of complications is greater than the number of patients with complications because some patients had multiple complications.

0.05) more likely to undergo craniotomy. Patients in the delayed intervention group had a significantly worse $(\mathrm{p}<$ $0.05)$ GOS score and mortality rate and a longer hospital stay.

\section{Discussion}

Patients with mTBI treated using an NSI are a small yet important part of the population. While various studies have suggested that between $0.7 \%$ and $8.7 \%$ of mTBI patients require NSI, the estimated incidence varies depending on operational definitions of mTBI and NSI as well as the time interval of analysis. ${ }^{1,2,6,7,9,10,20,22}$ Among 1688 adults with mTBI, we found the NSI rate to be $8.2 \%$, suggesting that the prevalence of mTBIs requiring NSI is at the higher end of rates reported in the literature. This could be related to our inclusion of patients on anticoagulants or antiplatelets, whereas most other studies excluded such patients. Additionally, our operational definition of NSI is broad and comprises the elevation of skull fractures and the placement of monitoring devices, unlike in other studies. If such patients are excluded, our NSI rate decreases to $6.3 \%(108 / 1688)$.

There has been little research on mTBI patients requiring NSI; therefore, little is known regarding their neurological outcomes, risk factors, morbidity, and mortality. Knowing the outcomes of mTBI patients requiring NSI is important to help guide patient counseling and the appropriate utilization of acute and rehabilitative resources. Our findings suggest that mTBI patients requiring NSI have higher mortality rates, worse outcomes, and longer hospital stays than those who do not require NSI.

We observed an in-hospital mortality rate of 13\% in patients with mTBI who required an NSI, which is higher than the very low reported rate for mTBIs $(0.3 \%-$ $1.8 \%)^{6,8,10}$ and between the mortality rates for moderate $(8 \%-10.4 \%)^{8,23}$ and severe $(28.7 \%-44.8 \%)^{8,23}$ TBIs. Moreover, according to the literature, mTBI patients with an abnormal head CT have a mortality rate of $3.2 \%$ at 2 weeks postdischarge, still markedly lower than our mortality rate in mTBI patients requiring an NSI. ${ }^{23}$
TABLE 5. Outcome of NSI in $139 \mathrm{mTBI}$ patients

\begin{tabular}{cc}
\hline \multicolumn{1}{c}{ Parameter } & No. \\
\hline Mean LOS in days & $16 \pm 31$ \\
\hline Mean GOS score & $3.6 \pm 1.2$ \\
\hline Discharge destination (\%) \\
\hline Home & $75(54)$ \\
\hline Rehabilitation & $41(29)$ \\
\hline Long-term care facility & $4(3)$ \\
\hline Morgue & $18(13)$ \\
\hline Other & $1(1)$ \\
\hline
\end{tabular}

Our study is the first, to our knowledge, to focus on the early neurological outcomes of patients with mTBI requiring NSI. Our results suggest that while $56 \%$ of these patients had an overall good outcome, $44 \%$ had a poor neurological outcome (defined as a GOS score $<4$ ). This proportion of poor outcomes is significantly higher than the $25 \%$ rate of poor outcomes reported in a multicenter study in an mTBI population. ${ }^{13}$ In addition, the mean LOS for patients in our study was $16 \pm 31$ days, which is greater than that for previously reported mTBI patients (mean 7 days ${ }^{13}$ and $9.2 \pm 11.4$ days $^{12}$ ) and approached the LOS for moderate and severe TBI $(17.2 \pm 20.1$ and $18.2 \pm 20.7$, respectively). ${ }^{12}$ Therefore, mTBI patients requiring NSI will require a much longer inpatient stay and higher resource allocation before they are ready for rehabilitation.

Huynh et al. suggested that patients presenting with a GCS score of 15 can be safely managed without a neurosurgical consult even if the head CT is abnormal given the absence of NSI in 56 such patients in their study. ${ }^{11}$ On the contrary, we found in our population that a majority (61\%) of mTBI patients with an NSI had presented to the emergency department with a GCS score of 15 . Only $12 \%$ had presented with a GCS score of 13 , and $27 \%$ had presented with a GCS of 14. Prior studies have conflicting data regarding whether a patient presenting with a GCS score of 13 has an increased likelihood of NSI compared with a patient presenting with a GCS score of 14 or $15 .{ }^{2,6,10,22}$ At our institution, we routinely obtain neurosurgical consults for patients with an intracerebral hemorrhage on head CT, regardless of whether their admission GCS score is 15 . This clinical protocol in our trauma center is supported by these study findings.

Our study analyzed predictors of a poor neurological outcome in an mTBI population warranting NSI. We found that increased age, anticoagulant or antiplatelet use, subarachnoid hematomas, subdural hematomas, postoperative complications, and delayed neurosurgical intervention were associated with an increased odds of a poor neurological outcome (GOS score < 4). Importantly, ISS was not associated with a poor outcome, whereas head AIS was. This demonstrates that the good and poor outcome groups had similar overall injury burdens, but those with more severe head injuries fared worse. Using multivariate logistic regression, we found that older age ( $>60$ years) was the only strong, independent predictor of a poor neurological outcome. We found that patients over the age of 60 years had a 6 -fold increase in the odds of having a poor 
TABLE 6. Comparison of patients with and without poor outcomes

\begin{tabular}{|c|c|c|c|c|}
\hline Factor & Good Outcome: GOS Score >3 $(n=78)$ & Poor Outcome: GOS Score <4 $(n=61)$ & OR $(95 \% \mathrm{Cl})$ & p Value \\
\hline Female & $9(12)$ & $13(21)$ & $2.1(0.8-5.2)$ & 0.117 \\
\hline Age $>60$ yrs & $3(4)$ & $22(36)$ & $14.1(4.0-50.1)$ & $0.000^{*}$ \\
\hline Intraparenchymal contusion & $39(50)$ & $35(57)$ & $1.4(0.7-2.6)$ & 0.387 \\
\hline Epidural hematoma & $44(56)$ & $10(16)$ & $0.2(0.1-0.3)$ & $0.000^{*}$ \\
\hline Subdural hematoma & $44(56)$ & $49(80)$ & $3.1(1.5-6.8)$ & $0.003^{*}$ \\
\hline Subarachnoid hematoma & $38(42)$ & $40(66)$ & $2.0(1.0-4.0)$ & $0.047^{*}$ \\
\hline Skull fracture & $65(83)$ & $32(53)$ & $0.2(0.1-0.5)$ & $0.000^{*}$ \\
\hline Midline shift >5 mm & $43(55)$ & $25(41)$ & $0.6(0.3-1.1)$ & 0.098 \\
\hline Intubated in emergency room & $29(37)$ & $25(41)$ & $1.2(0.6-2.3)$ & 0.648 \\
\hline Craniotomy & $64(82)$ & $40(66)$ & $0.4(0.2-0.9)$ & $0.026^{*}$ \\
\hline Insertion of monitor & $17(22)$ & $20(37)$ & $1.8(0.8-3.7)$ & 0.146 \\
\hline Postop complications present & $7(9)$ & $13(21)$ & $2.8(1.0-7.4)$ & $0.040^{*}$ \\
\hline Delayed intervention group & $11(14)$ & $21(34)$ & $3.2(1.4-7.3)$ & $0.005^{*}$ \\
\hline Antiplatelet or anticoagulant use & $1(1)$ & $10(16)$ & $15.2(1.9-125.0)$ & $0.001^{*}$ \\
\hline
\end{tabular}

$\mathrm{n}=$ number of patients.

* Statistically significant $(p<0.05)$.

outcome. Although there are no other reports on outcome predictors in mTBI patients requiring NSI, numerous studies in patients with mTBI and TBI support our findings. A subanalysis of 182 patients with mTBI among 235 patients with TBI in a multicenter prospective trial by Mosenthal et al. also revealed that elderly (age $>60$ years) patients with mTBI were more likely to be discharged with severe disability (GOS Score 3 ) than young patients (23\% vs $9 \%$, $\mathrm{p}=0.03) .{ }^{13} \mathrm{~A}$ retrospective analysis of TBI patients from the New York State Trauma Registry also suggested that despite higher GCS scores, lower ISSs, and lower New ISSs (NISSs) on presentation in elderly patients than in nonelderly patients, the elderly were discharged with lower mean GCS scores (14.6 vs 14.7, p < 0.01) and were twice as likely to be discharged to extended care facilities $(54 \%$ vs $26 \%$, p < 0.01). ${ }^{21}$ Murray et al.'s multivariable prognostic analysis of 8686 patients pooled from 8 randomized controlled trials and 3 observational studies suggested that age (OR 2.14) was one of the most powerful independent prognostic variables for a poor GOS score at 6 months postinjury in all TBI patients. ${ }^{14}$

Mild TBI patients in our study had a more favorable outcome (decreased odds of a poor neurological outcome) if they had undergone craniotomy, either immediately or delayed, or had epidural hematoma or skull fracture. However, the presence of an epidural hematoma was the only independent predictor of increased odds of a good outcome. This finding was also supported by a pooled analysis of 8 TBI studies, including mild, moderate, and severe TBI patients by Murray et al., in which epidural hematoma (OR 1.6) predicted a good GOS score at 6 months postinjury. ${ }^{14}$ Previous TBI studies have implicated subarachnoid hemorrhage and subdural hematoma as predictors of a poor outcome for TBI patients..$^{14,16,19}$ However, after cor-

TABLE 7. Results of multivariable logistic regression analysis predicting a poor outcome*

\begin{tabular}{lcccc}
\hline \multicolumn{1}{c}{ Factor } & Parameter Estimate & Standard Error & Adjusted OR (95\% Cl) & $\mathrm{p} \mathrm{Value}$ \\
\hline Sex & 0.7 & 0.6 & $2.0(0.6-6.8)$ & 0.253 \\
\hline Age $>$ 60 yrs & 2.17 & 0.8 & $6.0(1.4-27.0)$ & $0.019 \dagger$ \\
\hline Epidural hematoma & -1.2 & 0.5 & $0.3(0.1-0.7)$ & $0.009 \dagger$ \\
\hline Skull fracture & -0.7 & 0.5 & $0.5(0.2-1.3)$ & 0.149 \\
\hline Subarachnoid hematoma & 0.6 & 0.5 & $1.8(0.8-4.4)$ & 0.207 \\
\hline Postop complications present & 0.9 & 0.6 & $2.4(0.7-7.9)$ & 0.162 \\
\hline Subdural hematoma & 0.5 & 0.5 & $1.6(0.6-4.5)$ & 0.349 \\
\hline Midline shift $>5$ mm & 0.8 & 0.7 & $2.2(0.6-8.4)$ & 0.221 \\
\hline Craniotomy & -0.5 & 0.6 & $0.6(0.2-1.9)$ & 0.383 \\
\hline ICP monitor & 0.3 & 0.6 & $1.4(0.5-4.3)$ & 0.567 \\
\hline Antiplatelet or anticoagulant use & 1.6 & 1.3 & $4.7(0.4-62.0)$ & 0.237 \\
\hline Constant & -0.4 & 0.8 & 0.6 & \\
\hline
\end{tabular}

* Hosmer-Lemeshow chi-square goodness of fit test statistic was 4.164, $8 \mathrm{df}, p=0.842$.

† Statistically significant $(p<0.05)$. 
TABLE 8. Comparison of patients in the immediate and delayed intervention groups

\begin{tabular}{|c|c|c|c|}
\hline Parameter & Immediate Intervention ( $n=107)$ & Delayed Intervention $(n=32)$ & $\mathrm{p}$ Value \\
\hline \multicolumn{4}{|l|}{ Demographics } \\
\hline Mean age in yrs & $40.7 \pm 18.2$ & $47.5 \pm 18.1$ & 0.071 \\
\hline Female (\%) & $14(13)$ & $8(25)$ & 0.105 \\
\hline Mean ISS & $26.0 \pm 8.7$ & $27.1 \pm 7.6$ & 0.503 \\
\hline Mean head AIS & $4.4 \pm 0.6$ & $4.5 \pm 0.6$ & 0.484 \\
\hline Mean GCS score & $14.5 \pm 0.7$ & $14.5 \pm 0.8$ & 0.799 \\
\hline Antiplatelet or anticoagulant use (\%) & $6(6)$ & $5(16)$ & 0.066 \\
\hline \multicolumn{4}{|l|}{ Radiographic findings (\%) } \\
\hline Intracranial contusion & $6(6)$ & $3(9)$ & 0.447 \\
\hline Subarachnoid hematoma & $58(54)$ & $20(63)$ & 0.407 \\
\hline Epidural hematoma & $45(42)$ & $9(28)$ & 0.156 \\
\hline Subdural hematoma & $69(65)$ & $24(75)$ & 0.267 \\
\hline Skull fracture & $80(75)$ & $17(53)$ & $0.019^{*}$ \\
\hline Midline shift $>5 \mathrm{~mm}$ & $58(54)$ & $10(31)$ & $0.023^{*}$ \\
\hline \multicolumn{4}{|l|}{ Interventions } \\
\hline Craniotomy (\%) & $86(80)$ & $18(56)$ & $0.006^{*}$ \\
\hline ICP monitor (\%) & $25(23)$ & $12(38)$ & 0.112 \\
\hline \multicolumn{4}{|l|}{ Outcomes } \\
\hline NSI complication rate (\%) & $16(15)$ & $4(13)$ & 0.729 \\
\hline Mean LOS & $12.0 \pm 11.3$ & $30.2 \pm 58.9$ & $0.003^{*}$ \\
\hline Mean GOS score & $3.7 \pm 1.2$ & $2.9 \pm 1.3$ & $0.003^{*}$ \\
\hline Mortality rate (\%) & $10(9)$ & $8(25)$ & $0.021^{*}$ \\
\hline
\end{tabular}

rection for other covariates, our logistic regression analysis did not show these factors to be predictors of a poor outcome in this subpopulation. This finding may be explained by the coexisting variables (age, presence or absence of other types of intracerebral hemorrhage) or the inclusion criteria of our study (limited to mTBI requiring NSI).

In comparing the immediate and delayed intervention groups, we found no differences with regard to age, ISS, head AIS, admission GCS score, complication rate from NSI, or antiplatelet or anticoagulant use. However, the 2 groups significantly differed in outcomes. Specifically, the later intervention group had a longer LOS, lower GOS score, and higher mortality. Those in the immediate intervention group were more likely to undergo a craniotomy for surgical evacuation of a hematoma, whereas those in the delayed intervention group were more likely to receive an intracranial monitoring device related to delayed edema not amenable to surgical evacuation. Our results demonstrated that patients with a favorable outcome had a higher rate of craniotomy than those with an unfavorable outcome. Furthermore, if immediate and delayed intervention groups are compared after controlling for craniotomies, the delayed NSI group was no longer predictive of a poor neurological outcome. Patel et al. found no difference between early and late failure of nonoperative management in short-term outcomes (activities of daily living, mortality, and deficits). ${ }^{15}$ Notably, this was a study of 56 patients in whom early ( $<24$ hours) and late failure $(>24$ hours) was defined by time interval rather than in relation to the number of head CTs obtained prior to intervention. In the review by Patel et al., $26 \%$ of the patients in the late intervention group had a poor outcome, as compared with $16 \%$ of patients in the immediate intervention group. Chang et al. conducted a retrospective analysis of serial head CT scans and the clinical status of 113 patients with TBI to identify predictors of intraparenchymal hemorrhage progression. ${ }^{5}$ Nonoperative management failed in 15 patients (13\%), who subsequently required surgical evacuation. Multivariable logistic regression showed that 3 factors were independently associated with the failure of nonoperative management: worsened GCS score between the initial and follow-up head CT, increase in hematoma volume $\left(>5 \mathrm{~cm}^{3}\right)$, and effacement of cisterns on the initial head CT. It can be postulated that any of these factors contributed to the poor outcomes in the delayed NSI group, which obtained NSI after the failure of nonoperative management. Early literature stressed the importance of rapid surgical intervention in those requiring it, as Seelig et al. demonstrated that those with traumatic subdural hematoma who underwent surgery in the first 4 hours had a mortality rate of $30 \%$, as compared with a $90 \%$ mortality rate in those who underwent surgery after more than 4 hours. ${ }^{18}$

This study had several limitations: 1) a retrospective study design from a single institution, 2) moderate sample size (despite 10-year data collection), 3) lack of standardized blinded CT interpretations, 4) relatively short-term outcome evaluation, 5) no standard protocol for NSI, 6) definition of delayed intervention by the number of CTs 
rather than by time, and 7) nonisolated TBI patient population. However, the ISS and head AIS demonstrated that the majority of the injury burden is in the head region. Of the total ISS of $26,19.4$ points is accounted by the head AIS, meaning the other 2 regions had an average AIS of only 2 .

\section{Conclusions}

Our findings indicate that mTBI patients requiring NSI have worse outcomes, higher mortality rates, and longer LOSs than mTBI patients who do not require NSI. Therefore, mTBI patients receiving NSI should be classified as complicated cases of mTBI, separating them from medically managed mTBI patients. In patients with mTBI requiring NSI, an increased age has a 6-fold increase in the odds of a poor neurological outcome, whereas epidural hematoma increases the odds of a good neurological outcome. Patients in the delayed NSI group (in whom nonoperative management had failed) had significantly worse outcomes, higher mortality, and longer LOSs than patients in the immediate NSI group. Future prospective studies of mTBI patients receiving NSI are warranted to verify these conclusions, with a special focus on timing of the NSI, neurological deterioration, standardization of cranial CT analysis, and long-term outcome measures.

\section{References}

1. Bee TK, Magnotti LJ, Croce MA, Maish GO, Minard G, Schroeppel TJ, et al: Necessity of repeat head CT and ICU monitoring in patients with minimal brain injury. J Trauma 66:1015-1018, 2009

2. Borczuk P: Predictors of intracranial injury in patients with mild head trauma. Ann Emerg Med 25:731-736, 1995

3. Cameron JL, Cameron AM: Current Surgical Therapy, ed 10. Philadelphia: Elsevier Saunders, 2011

4. Cassidy JD, Carroll LJ, Peloso PM, Borg J, von Holst H, Holm L, et al: Incidence, risk factors and prevention of mild traumatic brain injury: results of the WHO Collaborating Centre Task Force on Mild Traumatic Brain Injury. J Rehabil Med 43 (43 Suppl):28-60, 2004

5. Chang EF, Meeker M, Holland MC: Acute traumatic intraparenchymal hemorrhage: risk factors for progression in the early post-injury period. Neurosurgery 61 (1 Suppl):222-231, 2007

6. Culotta VP, Sementilli ME, Gerold K, Watts CC: Clinicopathological heterogeneity in the classification of mild head injury. Neurosurgery 38:245-250, 1996

7. Dacey RG Jr, Alves WM, Rimel RW, Winn HR, Jane JA: Neurosurgical complications after apparently minor head injury. Assessment of risk in a series of 610 patients. J Neurosurg 65:203-210, 1986

8. Demetriades D, Kuncir E, Murray J, Velmahos GC, Rhee P, Chan L: Mortality prediction of head Abbreviated Injury Score and Glasgow Coma Scale: analysis of 7,764 head injuries. J Am Coll Surg 199:216-222, 2004

9. Haydel MJ, Preston CA, Mills TJ, Luber S, Blaudeau E, DeBlieux PM: Indications for computed tomography in patients with minor head injury. N Engl J Med 343:100-105, 2000

10. Hsiang JN, Yeung T, Yu AL, Poon WS: High-risk mild head injury. J Neurosurg 87:234-238, 1997

11. Huynh T, Jacobs DG, Dix S, Sing RF, Miles, WS, Thomason,
MH: Utility of neurosurgical consultation for mild traumatic brain injury. Am Surg 72:1162-1167, 2006

12. Kim YJ: The impact of time from ED arrival to surgery on mortality and hospital length of stay in patients with traumatic brain injury. J Emerg Nurs 37:328-333, 2011

13. Mosenthal AC, Livingston DH, Lavery RF, Knudson MM, Lee S, Morabito D, et al: The effect of age on functional outcome in mild traumatic brain injury: 6-month report of a prospective multicenter trial. J Trauma 56:1042-1048, 2004

14. Murray GD, Butcher I, McHugh GS, Lu J, Mushkudiani NA, Maas AI, et al: Multivariable prognostic analysis in traumatic brain injury: results from the IMPACT study. J Neurotrauma 24:329-337, 2007

15. Patel NY, Hoyt DB, Nakaji P, Marshall L, Holbrook T, Coimbra R, et al: Traumatic brain injury: patterns of failure of nonoperative management. J Trauma 48:367-375, 2000

16. Qu XD, Shrestha R, Wang MD: Risk factors analysis on traumatic brain injury prognosis. Chin Med Sci J 26:98-102, 2011

17. Rutland-Brown W, Langlois JA, Thomas KE, Xi YL: Incidence of traumatic brain injury in the United States, 2003. J Head Trauma Rehabil 21:544-548, 2006

18. Seelig JM, Becker DP, Miller JD, Greenberg RP, Ward JD, Choi SC: Traumatic acute subdural hematoma: major mortality reduction in comatose patients treated within four hours. N Engl J Med 304:1511-1518, 1981

19. Servadei F, Murray GD, Teasdale GM, Dearden M, Iannotti F, Lapierre F, et al: Traumatic subarachnoid hemorrhage: demographic and clinical study of 750 patients from the European brain injury consortium survey of head injuries. Neurosurgery 50:261-269, 2002

20. Stiell IG, Wells GA, Vandemheen K, Clement C, Lesiuk H, Laupacis A, et al: The Canadian CT Head Rule for patients with minor head injury. Lancet 357:1391-1396, 2001

21. Susman M, DiRusso SM, Sullivan T, Risucci D, Nealon P, Cuff S, et al: Traumatic brain injury in the elderly: increased mortality and worse functional outcome at discharge despite lower injury severity. J Trauma 53:219-224, 2002

22. Thiruppathy SP, Muthukumar N: Mild head injury: revisited. Acta Neurochir (Wien) 146:1075-1083, 2004

23. Timmons SD, Bee T, Webb S, Diaz-Arrastia RR, Hesdorffer D: Using the abbreviated injury severity and Glasgow Coma Scale scores to predict 2-week mortality after traumatic brain injury. J Trauma 71:1172-1178, 2011

\section{Disclosure}

Dr. Prestigiacomo is a consultant for Stryker, Thermopeutix, and Aesculap.

\section{Author Contributions}

Conception and design: Sifri. Acquisition of data: Tierney. Analysis and interpretation of data: Tierney, Sifri. Drafting the article: Tierney, Nayak, Sifri. Critically revising the article: all authors. Approved the final version of the manuscript on behalf of all authors: Tierney. Statistical analysis: Tierney. Administrative/ technical/material support: Sifri. Study supervision: Sifri.

\section{Correspondence}

Kevin Tierney, Rutgers New Jersey Medical School, Department of Emergency Medicine, 30 Bergen St., ADMC 1110, Newark, NJ 07103. email: kevin.tierney@gmail.com. 